\title{
Aldosterone nongenomically induces angiotensin II receptor dimerization in rat kidney: role of mineralocorticoid receptor and NADPH oxidase
}

\author{
Kittisak Sinphitukkul ${ }^{1}$, Krissanapong Manotham² ${ }^{2}$ Somchai Eiam-Ong ${ }^{3}$, Somchit Eiam-Ong ${ }^{4}$
}

\begin{abstract}
${ }^{1}$ Graduate Division, Faculty of Medicine, Chulalongkorn University, Bangkok, Thailand ${ }^{2}$ Molecular and Cell Biology Unit, Department of Medicine, Lerdsin General Hospital, Bangkok, Thailand

${ }^{3}$ Department of Medicine, Division of Nephrology, Faculty of Medicine, Chulalongkorn University, Bangkok, Thailand

${ }^{4}$ Department of Physiology, Faculty of Medicine, Chulalongkorn University, Bangkok, Thailand
\end{abstract}

Submitted: 30 March 2017

Accepted: 1 June 2017

Arch Med Sci 2019; 15 (6): 1589-1598

DOI: https://doi.org/10.5114/aoms.2019.87135

Copyright $\odot 2019$ Termedia \& Banach

\section{Abstract}

Introduction: Previous in vitro studies demonstrated that aldosterone nongenomically induces transglutaminase (TG) and reactive oxygen species (ROS), which enhanced angiotensin II receptor (ATR) dimerization. There are no in vivo data in the kidney.

Material and methods: Male Wistar rats were intraperitoneally injected with normal saline solution, or aldosterone (Aldo: $150 \mu \mathrm{g} / \mathrm{kg} \mathrm{BW}$ ); or received pretreatment with eplerenone (mineralocorticoid receptor (MR) blocker, Ep. + Aldo), or with apocynin (nicotinamide adenine dinucleotide phosphate (NADPH) oxidase inhibitor, Apo. + Aldo) $30 \mathrm{~min}$ before aldosterone. Thirty minutes after aldosterone injection, protein abundances of dimeric and monomeric forms of $A T_{1} R$ and $A T_{2} R$, and protein abundances and localizations of TG2 and p47phox, a cytosolic subunit of NADPH oxidase, were determined by Western blot analysis and immunohistochemistry, respectively.

Results: Protein abundances of dimeric forms of $A T_{1} R$ and $A T_{2} R$ were enhanced by $170 \%$ and $70 \%$, respectively. Apocynin could block dimeric forms of both receptors while eplerenone inhibited only $\mathrm{AT}_{2} \mathrm{R}$. Monomeric protein levels of both receptors were maintained. Aldosterone significantly enhanced TG2 and p47phox protein abundances, which were blunted by eplerenone or apocynin. Aldosterone stimulated p47phox protein expression in both the cortex and the medulla while TG2 was induced mostly in the medulla. Eplerenone or apocynin normalized the immunoreactivity of both TG2 and p47phox.

Conclusions: This is the first in vivo study demonstrating that aldosterone nongenomically increases renal TG2 and p47phox protein expression and then activates $A T_{1} R$ and $A T_{2} R$ dimerizations. Aldosterone-stimulated $A T_{1} R$ and $A T_{2} R$ dimerizations are mediated through activation of NADPH oxidase. Aldosterone-induced $A T_{1} R$ dimer formation is an MR-independent pathway, whereas the formation of $A T_{2} R$ dimer is modulated in an MR-dependent manner.

Key words: aldosterone, angiotensin II receptor dimerization, mineralocorticoid receptor, NADPH oxidase, nongenomic action, rat kidney.

\author{
Corresponding author: \\ Assoc. Prof. Somchit \\ Eiam-Ong PhD \\ Department \\ of Physiology \\ Faculty of Medicine \\ Chulalongkorn University \\ Bangkok 10330, Thailand \\ Phone: (662) 256-4267 \\ Fax: (662) 256-4267 \\ E-mail: eiamong@yahoo.com
}




\section{Introduction}

Aldosterone, the final component of the renin-angiotensin-aldosterone system, plays an essential role in regulation of blood pressure by maintaining electrolyte and water homeostasis $[1,2]$. The classical role of aldosterone occurs via a genomic mechanism [3]. The aldosterone-mineralocorticoid receptor (MR) complex will bind to its hormone-responsive elements within the nucleus and promotes the expression of target genes [3]. At present, several examinations on aldosterone have turned to nongenomic actions which present a rapid onset ( $\leq 30 \mathrm{~min}$ ) [4-7].

A previous in vitro study has revealed the nongenomic action of aldosterone in enhancing the formation of angiotensin II type 1 receptor $\left(A T_{1} R\right)$ dimerization [8]. $A T_{1} R$ dimerization is essential for functional consequences of G-protein activation of various physiologic conditions $[9,10]$. Moreover, increased AT $\mathrm{R}$ dimerization plays a contributory role in pathologic conditions including hypertension and atherosclerosis [11]. In cultured mouse mesenteric arterioles, aldosterone nongenomically stimulates the activity of intracellular transglutaminase 2 (TG2), resulting in enhanced $A T_{1} R$ dimerization [8]. TG2 is a catalytic enzyme which catalyzes post-translational modification of proteins by covalent bond formation between free amine groups [12]. Besides TG2, elevation of reactive oxygen species (ROS) has been shown to enhance $A T_{1} R$ dimerization in human embryonic kidney (HEK) cells [13]. In cultured porcine proximal tubular cells, aldosterone nongenomically activates NADPH oxidase by translocating p47phox from the cytosolic compartment to bind its membranous subunit at the plasma membrane and then generates ROS production [14]. In addition, it has been demonstrated that ROS rapidly stimulates TG2 activity in cultured Swiss 3 T3 fibroblast cells [15]. Taken together, it appears that MR, TG2, NADPH oxidase, p47phox, and ROS might play important roles in the nongenomic action of aldosterone on $\mathrm{AT}_{1} \mathrm{R}$ dimerization. Despite the above in vitro evidence, there are no available in vivo data. Moreover, no in vitro data are available regarding the nongenomic action of aldosterone on angiotensin II type 2 receptor ( $\mathrm{AT}_{2} \mathrm{R}$ ) dimerization.

To obtain in vivo data, the present study was conducted in the rat kidneys 30 min following treatment with normal saline solution, or aldosterone; or receiving pretreatment with eplerenone (MR blocker) or with apocynin (NADPH oxidase inhibitor) $30 \mathrm{~min}$ before aldosterone injection. Western blot analysis was performed to measure protein abundances of dimeric and monomeric forms of $A T_{1} R$ and $A T_{2} R$ as well as TG2 and p47phox. Immunohistochemistry was performed for localization of TG2 and p47phox proteins.

\section{Material and methods}

\section{Experimental design}

Male Wistar rats weighing 200-240 g (National Laboratory Animal Center, Mahidol University, Nakornpathom, Thailand) were given conventional housing and diet. All animal protocols were approved by the Ethics Committee of Research, Chulalongkorn University (Permit number IRB 7/57). Serum creatinine of each rat should be $<1 \mathrm{mg} / \mathrm{dl}$ $[16,17]$. The rats were divided into four groups ( $n=8$ /group): sham (normal saline solution; NSS: $0.5 \mathrm{ml} / \mathrm{kg}$ BW by intraperitoneal injection, i.p.); Aldo (aldosterone $150 \mu \mathrm{g} / \mathrm{kg} \mathrm{BW}$, diluted in NSS, i.p.; Sigma, St. Louis, MO, USA); or pretreatment with eplerenone (MR blocker; $15 \mathrm{mg} / \mathrm{kg}$ BW; diluted in dimethyl sulfoxide, i.p.; Sigma; Ep. + Aldo) or with apocynin (NADPH oxidase inhibitor; $5 \mathrm{mg}$ / kg, diluted in NSS, i.p.; Sigma; Apo. + Aldo) 30 min before aldosterone injection [16-19]. We used this aldosterone dose as previously performed in the studies of nongenomic action of aldosterone on the protein expression of upstream/downstream mediators $[6,16,17]$. Therefore, in the present investigation, we further examined the effect of this dose on protein expression of ATR dimerization, TG2, and p47phox.

On the date of the experiment, 30 min following injection of NSS or aldosterone, the rats were anesthetized with thiopental (100 mg/kg BW, i.p.). Kidneys were removed, and a half of each kidney was fixed in liquid nitrogen, and then stored at $-80^{\circ} \mathrm{C}$ until use for measurement of dimeric and monomeric forms of ATRs ( $A T_{1} R$ and $A T_{2} R$ ), TG2, and 47phox protein abundances by Western blot analysis. The other half of renal tissue was fixed in $10 \%$ paraformaldehyde for localization of TG2 and $\mathrm{p} 47$ phox proteins by immunohistochemistry $[6,16,17]$.

\section{Western blot analysis}

The renal tissue samples were homogenized on ice with a homogenizer (T25 Basic, IKA, Selangor, Malaysia) in homogenizing buffer ( $20 \mathrm{mM}$ Tris- $\mathrm{HCl}$; pH 7.5, $2 \mathrm{mM} \mathrm{MgCl}, 0.2 \mathrm{M}$ sucrose, and $5 \%(\mathrm{v} / \mathrm{v})$ protease inhibitor cocktail (Sigma)). To get rid of crude debris, the kidney homogenates were centrifuged at 4,000 g (Sorvall Legend X1R, Thermo Fisher Scientific, Rockford, IL, USA) for $20 \mathrm{~min}$ at $4^{\circ} \mathrm{C}$. To harvest plasma membrane, the supernatant was further centrifuged at $17,000 \mathrm{~g}$ for $20 \mathrm{~min}$ at $4^{\circ} \mathrm{C}$ [20]. The pellet was dissolved in buffer. Total protein concentration was measured with Bradford protein assay reagent (Pierce, Rockford, IL, USA) following the manufacturer's protocol. The measurement of protein abundance was performed as previously described $[6,16,17]$. Proteins were resolved on $8 \%$ sodium dodecyl 
sulfate polyacrylamide gel electrophoresis for $A T_{1} R, A T_{2} R$ (dimeric and monomeric forms), TG2, p47phox, $\beta$-actin, and blotted onto nitrocellulose membrane (Bio-Rad Laboratories, Hercules, CA, USA). The membranes were incubated with primary monoclonal antibody to $\mathrm{AT}_{1} \mathrm{R}$ (1E10-1A9: SC-81671; 1 : 200; Santa Cruz Biotechnology, Dallas, TX, USA), AT 2 R (C-18: sc-7420; 1 : 300; Santa Cruz Biotechnology), TG2 (TG100: MA5-12915; 1 : 1,000; Thermo Fisher Scientific), p47phox (D-10: sc-17845; 1 : 250; Santa Cruz Biotechnology), or to $\beta$-actin (C4: sc-47778; 1 : 2,000; Santa Cruz Biotechnology), followed by the respective horseradish peroxidase-linked secondary antibody (Bio-Rad Laboratories). Immunoreactive proteins were detected by chemiluminescence detection (SuperSignal West Pico kit; Pierce) and documented by using a Molecular imager ChemiDoc XRS system (Bio-Rad Laboratories). Relative protein levels of $A T_{1} R$ and $A T_{2} R$ (dimeric and monomeric forms), TG2, and p47phox in each sample were presented as a percentage of the control normalized to its $\beta$-actin content.

\section{Immunohistochemical study}

Detection of protein localization was performed as previously described $[6,16,17]$. Paraffin-embedded kidney sections were cut into $4 \mu \mathrm{m}$ thick slices. The slides were deparaffinized and endogenous peroxidase was blocked by treatment with $3 \% \mathrm{H}_{2} \mathrm{O}_{2}$. The sections were incubated with the primary antibody TG2 (1 : 20,000; Thermo Fisher Scientific), or p47phox (1: 150; Santa Cruz Biotechnology) at $4^{\circ} \mathrm{C}$ overnight, followed by the respective horseradish peroxidase-linked

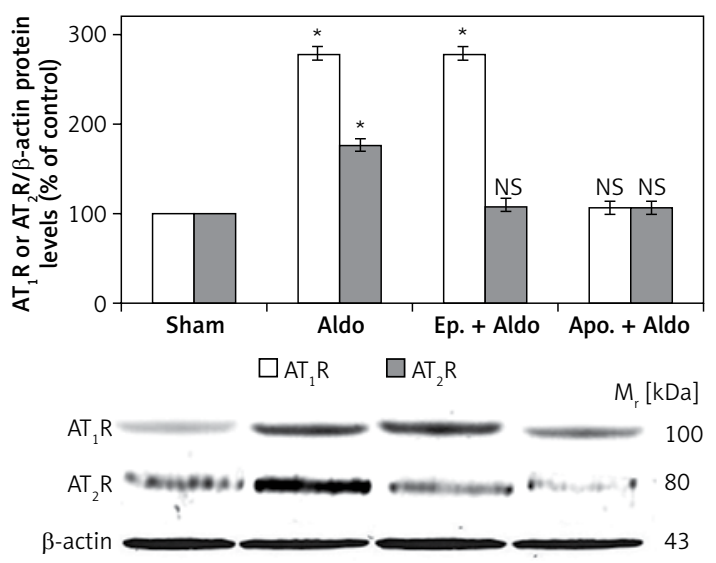

Figure 1. Western blot analysis of renal dimeric forms of $A T_{1} R$ and $A T_{2} R$ protein abundances in sham, Aldo, Ep. + Aldo, and Apo. + Aldo groups. Histogram bars show the densitometric analyses ratios of $A T_{1} R$, or $A T_{2} R$ to $\beta$-actin intensity, and the representative immunoblot photographs are presented

Data are means $\pm S D$ of 8 independent experiments. ${ }^{*} P<0.001$ compared with the respective sham group. secondary antibody (Bio-Rad Laboratories), then reacted with 3,3'-diaminobenzidine solution (Sig$\mathrm{ma}$ ). Three pathologists independently scored the staining intensity on a semi-quantitative fivetiered grading scale from 0 to $4(0=$ negative; 1 = trace; 2 = weak; 3 = moderate; 4 = strong) as previously described $[6,16,17]$.

\section{Statistical analysis}

Results of renal $A T_{1} R$ and $A T_{2} R$ (dimeric and monomeric forms), TG2, and p47phox protein abundances were expressed as mean \pm SD. Statistical differences between the groups were assessed by ANOVA (analysis of variance) with post-hoc comparison by Tukey's test where appropriate. A p-value $<0.05$ was considered statistically significant. Statistical tests were analyzed using the SPSS program version 22.0 (IBM Corp. Chicago, IL, USA). The median staining intensity (score) of renal TG2 and p47phox protein expression was presented as previously described $[6,16,17]$.

\section{Results}

\section{Aldosterone enhances renal ATRs dimer protein abundances}

The protein levels of $A T_{1} R$ and $A T_{2} R$ were assessed by Western blot analysis (Figure 1). Aldosterone enhanced protein abundances of dimeric forms of $A T_{1} R(100 \mathrm{kDa})$ and $A T_{2} R(80 \mathrm{kDa})$ from sham $(100 \%)$ to be $278 \pm 7 \%$ and $175 \pm 9 \%$, respectively $(p<0.001)$. Apocynin could block aldosterone-induced dimeric protein abundances of both AT R $(105 \pm 4 \%, p=0.12)$ and AT $_{2} \mathrm{R}(103 \pm 5 \%$, $p=0.57)$. Eplerenone had an inhibitory effect only

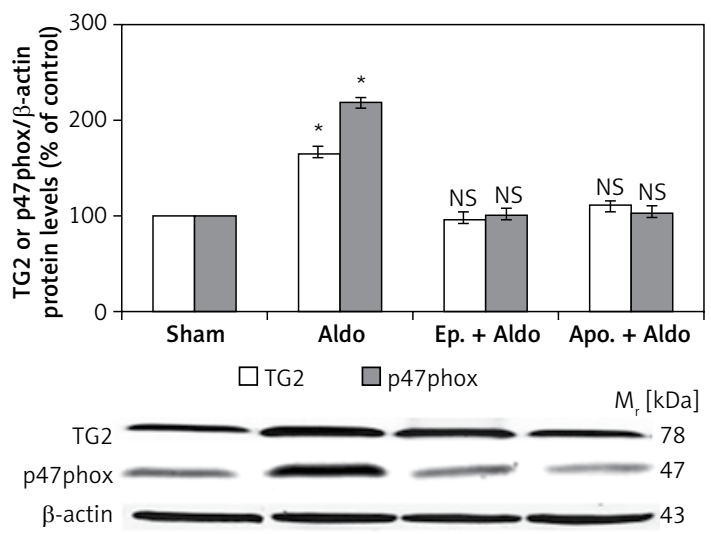

Figure 2. Western blot analysis of renal TG2 and p47phox protein abundances in sham, Aldo, Ep. + Aldo, and Apo. + Aldo groups. Histogram bars show the densitometric analyses ratios of TG2, or p47phox to $\beta$-actin intensity, and the representative immunoblot photographs are presented

Data are means $\pm S D$ of 8 independent experiments. ${ }^{*} P<0.001$ compared with the respective sham group. 
on dimeric forms of $\mathrm{AT}_{2} \mathrm{R}$ protein $(108 \pm 6 \%, p=$ 0.23). The dimeric protein levels of $A T_{1} R$ were still enhanced to be $276 \pm 8 \%(p<0.001)$ in the presence of MR blocker. Aldosterone did not alter protein levels of monomeric forms of $A T_{1} R(50 \mathrm{kDa})$ or $\mathrm{AT}_{2} \mathrm{R}(41 \mathrm{kDa})$ (data not shown).

\section{Aldosterone stimulates renal transglutaminase 2 (TG2) and p47phox protein abundances}

The protein levels of TG2 (78 kDa) and p47phox $(47 \mathrm{kDa})$ in the rat kidney were measured by Western blot analysis (Figure 2). Aldosterone significantly elevated protein abundances of TG2 and p47phox from sham (100\%) to be $165 \pm 8 \%$ and $218 \pm 11 \%$, respectively ( $p<0.001)$. Both eplerenone and apocynin, when each was used, completely abolished the effect of aldosterone-induced TG2 (97 $\pm 5 \%, p=0.48 ; 110 \pm 10 \%, p=0.40)$ and p47phox (101 $\pm 5 \%, p=0.90 ; 104 \pm 4 \%, p=$ $0.40)$, respectively.

\section{Renal TG2 protein expression is activated by aldosterone}

Protein expression of TG 2 in the cortex of sham is shown in Figure $3 \mathrm{~A}$ and Table I. The expression was grade 1 (trace) in the glomerulus, whereas the intensity was grade 4 (strong) in the peritubular capillary (Pcap). The immunostaining was moderately diffuse in the proximal convoluted tubule $(P C T)$. No staining was noted in the distal convoluted tubule (DCT) or cortical collecting duct (CCD). Aldosterone did not alter the intensity score in these areas (Figure 3 B). Interestingly, aldosterone induced the translocation of TG2 protein expression from the cytosol to the luminal membrane of PCTs. Eplerenone or apocynin pretreatment could blunt the effect induced by aldosterone (Figures 3 $C, D)$. The staining in the PCT returned to the same pattern as the sham.

In the outer medulla, aldosterone elevated the intensity score in all studied areas (Figure 3 F). Eplerenone or apocynin could lessen the immunoreactivity induced by aldosterone in the thick ascending limb of Henle's loop (TALH), vasa recta (VR) and thin limb of Henle's loop (tLH) (Figures $3 \mathrm{G}, \mathrm{H}$ ). The expression in the medullary collecting duct (MCD) remained. In the inner medulla, aldosterone enhanced the immunoreactivity in all studied regions (Figure $3 \mathrm{~J}$ ). Both eplerenone and apocynin, when each was used, could inhibit the effect of aldosterone on TG2 expression (Figures $3 \mathrm{~K}$, L; Table I).

\section{Renal p47phox protein expression is induced by aldosterone}

As shown in Figure 4 and Table I, the immunostaining of $p 47$ phox protein was obvious at the basolateral membrane of the renal tubules in both the cortex and the medulla. Furthermore, the expression was also well noted in the renal vasculature. In the cortex (Figure 4 B), aldosterone markedly enhanced the expression to be strong in the glomerulus, PCT, DCT and Pcap while the intensity in the CCD was grade 3 (moderate). Pretreatment with apocynin showed a greater inhibitory effect than eplerenone on aldosterone-induced p47phox expression (Figures $4 \mathrm{D}, \mathrm{C}$ respectively).

In the outer medulla, aldosterone elevated the intensity score in TALH and tLH (Figure 4 F). Eplerenone or apocynin could decrease the immunoreactivity in both areas (Figures $4 \mathrm{G}, \mathrm{H}$ ). The expression in the MCD and VR remained. In the inner medulla, aldosterone enhanced the immunoreactivity in all studied regions (Figure $4 \mathrm{~J}$ ). Eplerenone pretreatment had a greater blocking effect than apocynin (Figures 4 K, L; Table I).

\section{Discussion}

To our knowledge, these are the first in vivo data simultaneously demonstrating that the renal protein abundances of dimeric forms of $A T_{1} R$ and $A T_{2} R$ are enhanced 30 min following aldosterone administration (Figure 1). AT $R$ dimerization is important for functional consequences of G-protein activation [9]. Previous studies regarding $A T_{1} R$ found that $A T_{1} R$ dimerization influences receptor activation mechanisms including agonist/antagonist affinity, efficacy, trafficking, and specificity of signal transduction mediators [9, 10]. Under physiologic conditions, $\mathrm{AT}_{1} \mathrm{R}$ dimerization promoted sodium reabsorption in microdissected rat proximal tubule by stimulating $\mathrm{Na}^{+}$-ATPase activity [10]. In pathologic conditions, an increased $\mathrm{AT}_{1} \mathrm{R}$ dimerization was reported in monocytes isolated from hypertensive patients [11]. Moreover, monocytes of ApoE knockout mice exhibit high AT $_{1} \mathrm{R}$ dimer levels which cause atherosclerosis [11]. Indeed, elevation of $\mathrm{AT}_{2} \mathrm{R}$ dimerization was found in the renal cortex from a preeclampsia rat model [21].

In the present study, aldosterone enhanced $A T_{1} R$ dimers but did not alter AT $\mathrm{R}$ monomers. A previous study in vascular smooth muscle cells also showed that aldosterone increases $A T_{1} R$ dimers while the monomers are unchanged [8]. No data of $A T_{2} R$ dimerization related to aldosterone were reported in that study. The mechanism of this phenomenon remains to be established. ATRs belong to the seven membrane classes of G-protein-coupled receptors (GPCRs) which can dimerize in the endoplasmic reticulum (ER) [22]. A recent study in HEK cells demonstrated that corticotropic-releasing factor receptor type 1, a member of GPCRs, is assembled as dimers in the ER and transported to the plasma membrane [23]. Therefore, it is likely that aldosterone might enhance both $A T_{1} R$ 

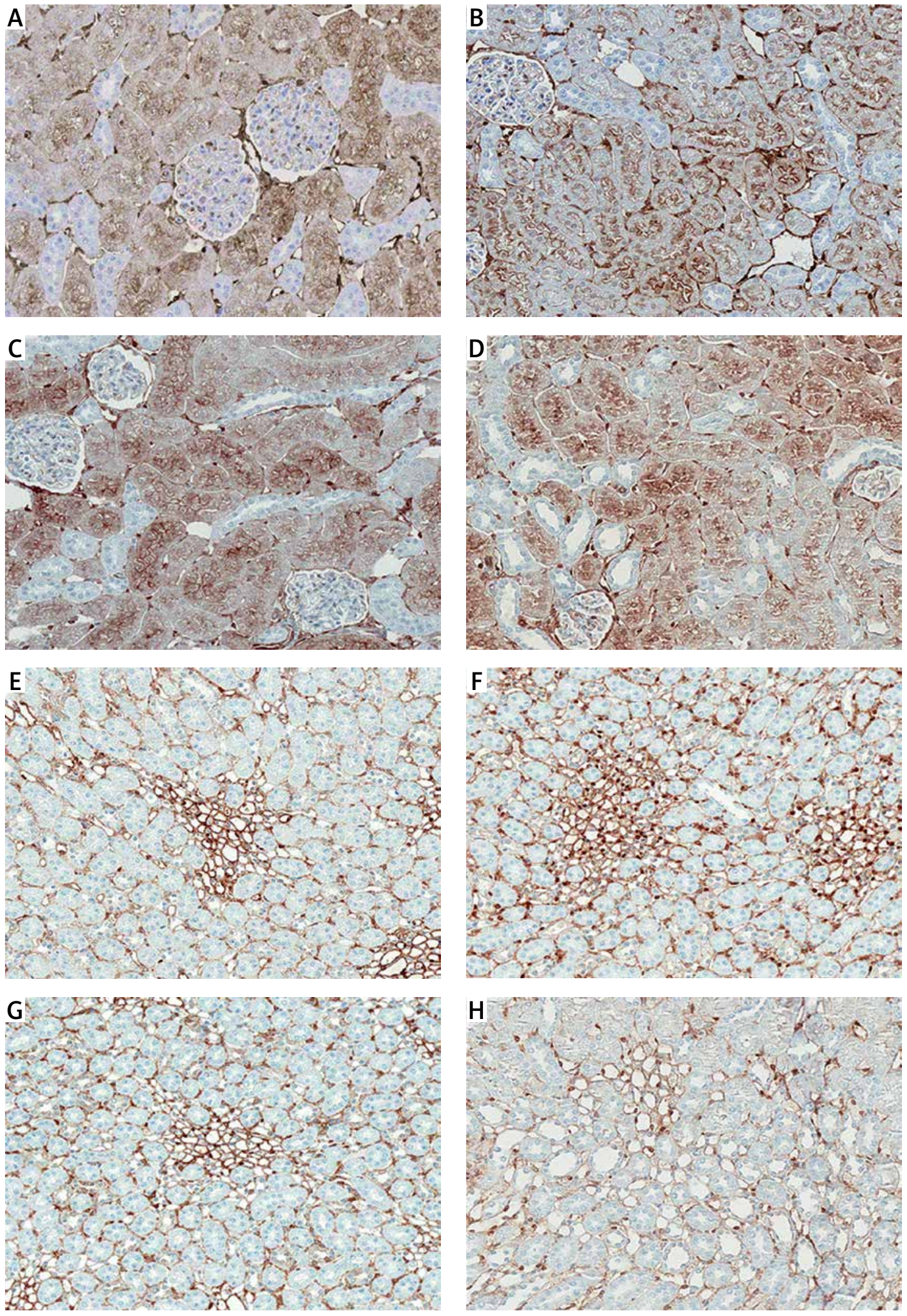

Figure 3. Representative immunohistochemical staining micrographs of renal TG2 protein expression in the cortex $(A-D)$, the outer medulla $(E-H)$, and the inner medulla (I-L) from sham (A, E, I), Aldo (B, F, J), Ep. + Aldo (C, G, K), and Apo. + Aldo (D, H, L). Original magnification 400× (A-D) and 200× (E-L) 

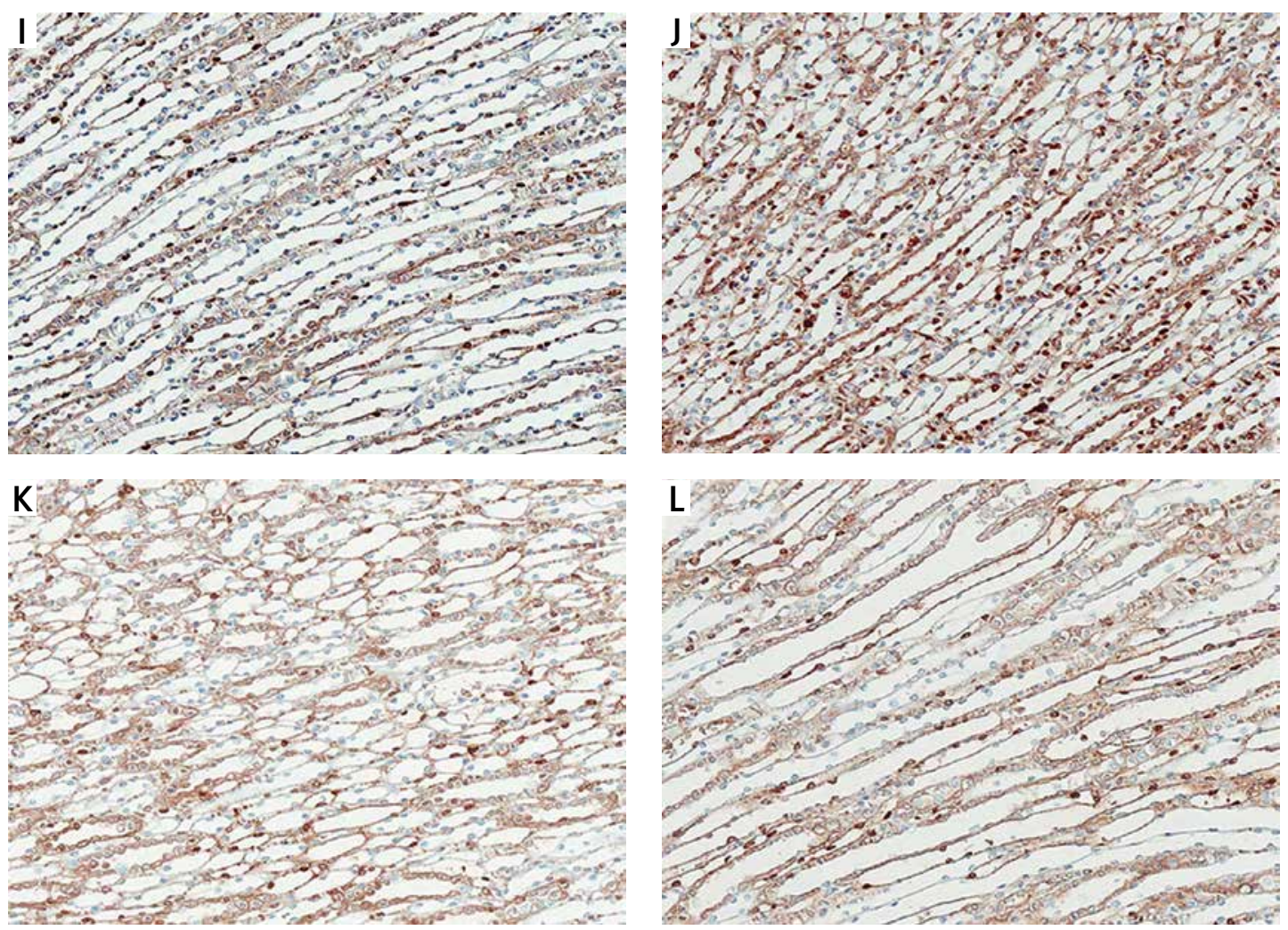

Figure 3. Cont.

Table I. Median staining intensity (score) of renal TG2 and p47phox protein expressions

\begin{tabular}{|c|c|c|c|c|c|c|c|c|}
\hline \multirow[t]{3}{*}{ Parameter } & \multicolumn{8}{|c|}{ Median staining intensity (score) } \\
\hline & \multicolumn{4}{|c|}{ TG2 } & \multicolumn{4}{|c|}{ p47phox } \\
\hline & Sham & Aldo & Ep. + Aldo & Apo. + Aldo & Sham & Aldo & Ep. + Aldo & Apo. + Aldo \\
\hline \multicolumn{9}{|l|}{ Cortex: } \\
\hline Glomerulus & 1 & 1 & 1 & 1 & 2 & 4 & 2 & 1 \\
\hline PCT & 3 & 3 & 3 & 3 & 2 & 4 & 3 & 2 \\
\hline $\mathrm{DCT}$ & 0 & 0 & 0 & 0 & 2 & 4 & 3 & 2 \\
\hline CCD & 0 & 0 & 0 & 0 & 1 & 3 & 1 & 1 \\
\hline Pcap & 4 & 4 & 4 & 4 & 2 & 4 & 3 & 2 \\
\hline \multicolumn{9}{|l|}{ Outer medulla: } \\
\hline TALH & 0 & 1 & 0 & 0 & 1 & 2 & 1 & 1 \\
\hline MCD & 0 & 1 & 1 & 1 & 1 & 1 & 1 & 1 \\
\hline VR & 3 & 4 & 3 & 1 & 4 & 4 & 4 & 4 \\
\hline $\mathrm{tLH}$ & 2 & 3 & 2 & 1 & 1 & 3 & 2 & 1 \\
\hline \multicolumn{9}{|l|}{ Inner medulla: } \\
\hline$M C D$ & 3 & 4 & 2 & 3 & 1 & 4 & 1 & 3 \\
\hline VR & 2 & 3 & 2 & 2 & 3 & 4 & 3 & 3 \\
\hline $\mathrm{tLH}$ & 1 & 3 & 1 & 1 & 2 & 4 & 2 & 3 \\
\hline
\end{tabular}

Staining intensity: $0=$ negative, no reactivity; $1=$ trace, faint or pale brown staining with less membrane reactivity; 2 = weak, light brown staining with incomplete membrane reactivity; 3 = moderate, shaded of brown staining of intermediate darkness with usually almost complete membrane reactivity; 4 = strong, dark brown to black staining with usually complete membrane pattern, producing a thick outline of the cell $[6,16,17]$. PCT - proximal convoluted tubule, DCT - distal convoluted tubule, CCD - cortical collecting duct, Pcap peritubular capillary, TALH - thick ascending limb of Henle's loop, MCD - medullary collecting duct, VR - vasa recta, $t L H$ - thin limb of Henle's loop. 

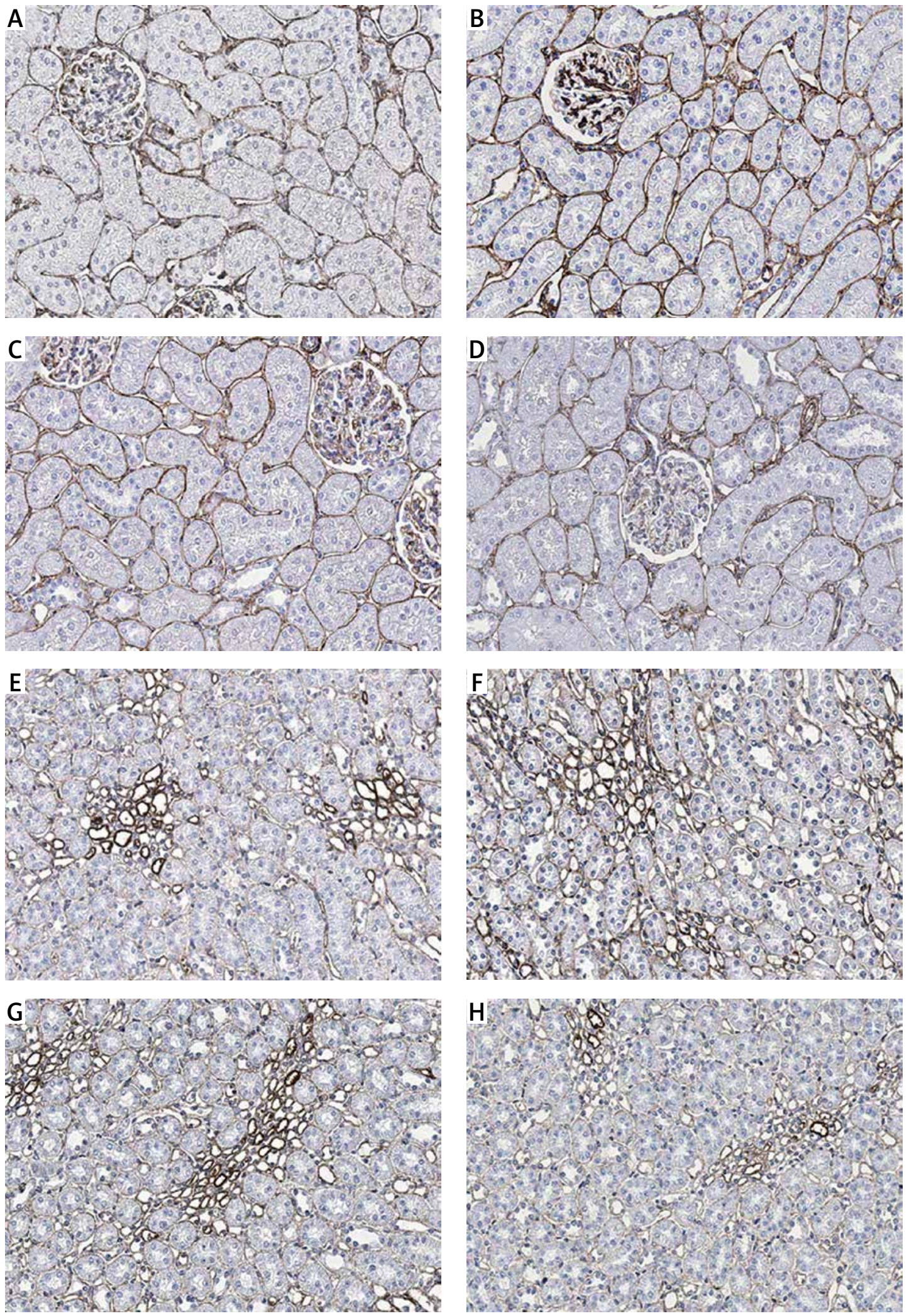

Figure 4. Representative immunohistochemical staining micrographs of renal p47phox protein expression in the cortex (A-D), the outer medulla (E-H), and the inner medulla (I-L) from sham (A, E, I), Aldo (B, F, J), Ep. + Aldo (C, $\mathrm{G}, \mathrm{K})$, and Apo. + Aldo (D, H, L). Original magnification 400× (A-D) and 200× (E-L) 

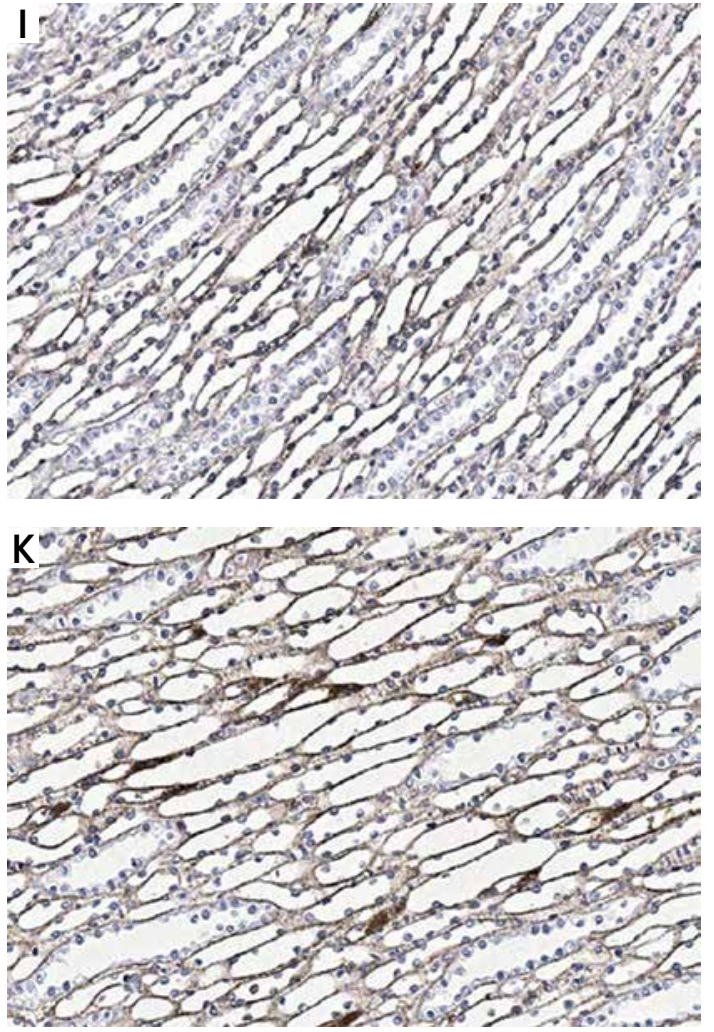

Figure 4. Cont.

and $\mathrm{AT}_{2} \mathrm{R}$ dimerizations within the $\mathrm{ER}$ and these dimers are transported to the plasma membrane, resulting in unchanged monomers.

This study is the first to demonstrate that aldosterone-induced AT $R$ dimerization occurs via an MR-independent pathway while aldosterone-induced $A T_{2} R$ dimerization is dependent on the MR pathway (Figure 1). Regardless of the MR pathway dependence or not, the enhancements of $A T_{1} R$ and $A T_{2} R$ dimerizations were suppressed by apocynin (Figure 1), indicating the mechanistic role of NADPH oxidase in stimulating dimerization of both ATRs.

As stated earlier, previous in vitro studies in various tissues suggested that there might be an interrelationship among NADPH oxidase, p47phox, ROS, and TG2 in contributing to the nongenomic stimulating action of aldosterone on $A T_{1}$ R dimerization. In support of this contention, the present study illustrates that aldosterone rapidly stimulates TG2 and p47phox, both of which are inhibited by apocynin (Figure 2). Therefore, in the MR-independent pathway, aldosterone might activate ROS via NADPH oxidase and then stimulate both TG2 and $A T_{1} R$ dimerization in the kidney.

Regarding the mechanism of $A \mathrm{~T}_{2} \mathrm{R}$ dimerization, in the present study, aldosterone elevated TG2 protein abundance via MR (Figure 2). A previous study reported that TG2 mediates cross-linking of $A T_{2} R$ into oligomers in HEK cells [24]. Howev-
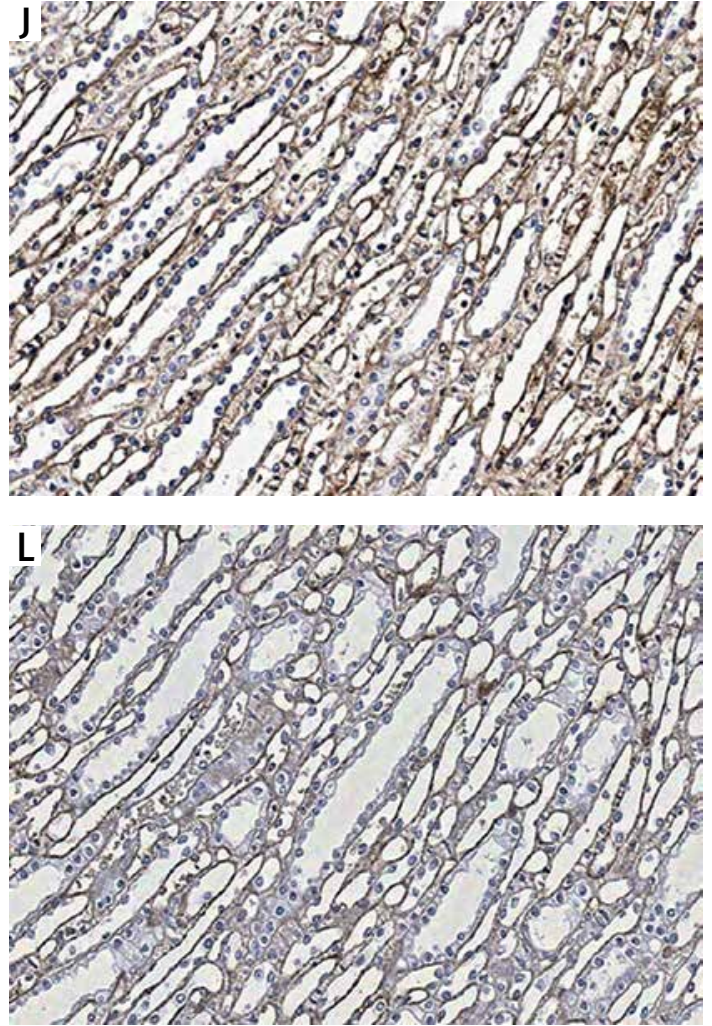

er, TG2-induced $\mathrm{AT}_{2} \mathrm{R}$ dimerization has not been shown in any studies. It is likely that aldosterone activates TG2 and then stimulates $\mathrm{AT}_{2} \mathrm{R}$ dimerization. One attractive effect of aldosterone on $A T_{2} R$ dimerization is enhancing ROS, since translocation of p47phox into the plasma membrane represents activation of NADPH oxidase and then leads to generation of $\operatorname{ROS}[25,26]$. The present study is the first to demonstrate that the nongenomic action of aldosterone on $\mathrm{AT}_{2} \mathrm{R}$ dimerization elevates plasma membrane protein abundance of p47phox by an MR-dependent mechanism (Figure 2). A former study reported that aldosterone nongenomically reduces cytosolic p47phox but the effect of plasma membrane $\mathrm{p} 47$ phox on $\mathrm{AT}_{2} \mathrm{R}$ dimerization was not examined [14]. Our data showed that aldosterone-induced $\mathrm{AT}_{2} \mathrm{R}$ dimerization is abolished by apocynin (Figure 1). The results suggest that aldosterone-induced ROS production from activation of NADPH oxidase has a potential role in $A T_{2} R$ dimerization.

Immunohistochemistry studies revealed that TG2 in the sham group showed weak and diffuse immunostaining in glomeruli and tubules (Figures $3 \mathrm{~A}, \mathrm{E}, \mathrm{I}$ ). Previous studies in normal rat kidney also revealed the similar baseline regional distribution of TG2 protein as in the present investigation [27, 28]. In the present study, aldosterone stimulated the expression of TG2 mostly in the medulla area (Figures $3 \mathrm{~F}, \mathrm{~J}$ ). Although the expression levels were 
not altered in the cortex, aldosterone nongenomically translocated the TG2 protein from the cytosol to the luminal membrane while eplerenone or apocynin could normalize the immunoreactivity (Figures 3 A-D). A previous study demonstrated that both $A T_{1} R$ and $A T_{2} R$ are highly abundant in the luminal membrane of renal tubular cells, for example in the proximal tubules and medullary TALH $[29,30]$. As such, aldosterone-induced TG2 might play a significant role in dimerization of $A T_{1} R$ or $A T_{2} R$ to regulate ion transport in the renal tubules. Moreover, the prominent TG2 expression was present in Pcap and VR by aldosterone (Table I). This implies some important effects of TG2 on these renal vascular functions. Of note, enhanced TG2 expression in the kidney has been related to the development of kidney diseases. TG2 protein abundance, activity, and expression were enhanced in the puromycin aminonucleoside-injection-induced experimental rat model of focal segmental glomerulosclerosis, subtotal nephrectomy-induced renal fibrosis, IgA nephropathy, and the rat renal transplantation model of chronic allograft nephropathy [27, 28, 31, 32]. Furthermore, TG inhibition could ameliorate experimental diabetic nephropathy, reduce fibrosis and preserve function in experimental chronic kidney disease [33-36].

For p47phox protein localization, immunostaining in the sham group was present in the glomeruli and renal vasculature in both the cortex and the medulla (Figures $4 \mathrm{~A}, \mathrm{E}, \mathrm{I}$ ). This baseline regional distribution of $\mathrm{p} 47 \mathrm{phox}$ protein is similar to previous studies in normal rat kidney [37-39]. Of interest, the present study shows more obvious staining at the basolateral membrane of renal tubules than those previously reported [37-39]. This discrepancy may be due to the more specific (monoclonal) p47phox antibody used in the present study. Aldosterone enhanced p47phox protein expression at the basolateral membrane in both the cortex and the medulla and this enhancement was normalized by eplerenone or apocynin (Figure 4). Interestingly, induction of renal p47phox protein expression has been implicated in some renal diseases such as diabetic nephropathy and nephrolithiasis $[37,38]$. Deletion of $p 47$ phox could attenuate the progression of kidney fibrosis and reduce albuminuria in diabetic nephropathy and nondiabetes-mediated glomerular injury [40, 41].

In conclusion, to our knowledge, this is the first in vivo study demonstrating that aldosterone nongenomically increases renal TG2 and p47phox protein expression and then activates $A T_{1} R$ and $A T_{2} R$ dimerizations. Aldosterone-stimulated $A T_{1} R$ and $A T_{2} R$ dimerizations are mediated through activation of NADPH oxidase. Aldosterone-induced $A T_{1} R$ dimer formation is an MR-independent path- way, whereas the formation of $\mathrm{AT}_{2} \mathrm{R}$ dimer is modulated in an MR-dependent manner.

\section{Acknowledgments}

This study was supported by (1) the Royal Golden Jubilee Ph.D. scholarship (grant No. PHD/ 0104/ 2556) awarded to Mr. Kittisak Sinphitukkul by the Thailand Research Fund under the Office of the Prime Minister, the Royal Thai Government 2014, and (2) the $90^{\text {th }}$ Anniversary of Chulalongkorn University Fund and Ratchadapiseksomphot Endowment Fund (No. 34 code: GCUGR1125572042D) by the Faculty of Medicine, Chulalongkorn University.

\section{Conflict of interest}

The authors declare no conflict of interest.

\section{References}

1. Connell JM, Davies E. The new biology of aldosterone. J Endocrinol 2005; 186: 1-20.

2. Aronow WS. Update of treatment of heart failure with reduction of left ventricular ejection fraction. Arch Med Sci Atheroscler Dis 2016; 1: e106-16.

3. Rozansky DJ. The role of aldosterone in renal sodium transport. Semin Nephrol 2006; 26: 173-81.

4. Thomas W, Harvey BJ. Mechanisms underlying rapid aldosterone effects in the kidney. Annu Rev Physiol 2011; 73: 335-57.

5. Winter C, Schulz N, Giebisch G, Geibel JP, Wagner CA. Nongenomic stimulation of vacuolar $\mathrm{H}^{+}$-ATPases in intercalated renal tubule cells by aldosterone. Proc Natl Acad Sci USA 2004; 101: 2636-41.

6. Eiam-Ong S, Sinphitukkul K, Manotham K, Eiam-Ong S. Rapid action of aldosterone onprotein expressions of protein kinase $C$ alpha and alphal sodium potassium adenosine triphosphatase in rat kidney. J Steriods Hormon Sci 2017; 2017: 2975853.

7. Vinson GP, Coghlan JP. Expanding view of aldosterone action, with an emphasis on rapid action. Clin Exp Pharmacol Physiol 2010; 37: 410-6.

8. Yamada M, Kushibiki M, Osanai T, Tomita H, Okumura K. Vasoconstrictor effect of aldosterone via angiotensin II type $1\left(\mathrm{AT}_{1}\right)$ receptor: possible role of $\mathrm{AT}_{1}$ receptor dimerization. Cardiovasc Res 2008; 79: 169-78.

9. Lyngso C, Erikstrup N, Hansen JL. Functional interactions between 7TM receptors in the renin-angiotensin system-dimerization or crosstalk? Mol Cell Endocrinol 2009; 302: 203-12.

10. Dias J, Ferrao FM, Axelband F, Carmona AK, Lara LS, Vieyra A. ANG-(3-4) inhibits renal $\mathrm{Na}^{+}$-ATPase in hypertensive rats through a mechanism that involves dissociation of ANG II receptors, heterodimers, and PKA. Am J Physiol 2014; 306: F855-63.

11. AbdAlla S, Lother H, Langer A, el Faramawy Y, Quitterer U. Factor XIIla transglutaminase crosslinks $\mathrm{AT}_{1}$ receptor dimers of monocytes at the onset of atherosclerosis. Cell 2004; 119: 343-54.

12. Eckert RL, Kaartinen MT, Nurminskaya M, et al. Transglutaminase regulation of cell function. Physiol Rev 2014; 94: 383-417.

13. AbdAlla S, Lother H, el Massiery A, Quitterer U. Increased $\mathrm{AT}_{1}$ receptor heterodimers in preeclampsia mediate en- 
hanced angiotensin II responsiveness. Nat Med 2001; 7: 1003-9.

14. Queisser N, Schupp N, Stopper H, Schinzel R, Oteiza PI. Aldosterone increases kidney tubule cell oxidants through calcium-mediated activation of NADPH oxidase and nitric oxide synthase. Free Radic Biol Med 2011; 51 : 1996-2006.

15. Lee ZW, Kwon SM, Kim SW, Yi SJ, Kim YM, Ha KS. Activation of in situ tissue transglutaminase by intracellular reactive oxygen species. Biochem Biophys Res Commun 2003; 305: 633-40.

16. Sinphitukkul K, Eiam-Ong S, Manotham K, Eiam-Ong S Nongenomic effects of aldosterone on renal protein expressions of $\mathrm{pEGFR}$ and $\mathrm{pERK} 1 / 2$ in rat kidney. Am J Nephrol 2011; 33: 111-20.

17. Eiam-Ong S, Sinphitukkul K, Manotham K, Eiam-Ong S. Rapid nongenomic action of aldosterone on protein expressions of Hsp90(alpha and beta) and pc-src in rat kidney. Biomed Res Int 2013; 2013: 346480.

18. Cook CS, Zhang L, Ames GB, Fischer J, Zhang J, Levin S. Single- and repeated-dose pharmacokinetics of eplerenone, a selective aldosterone receptor blocker, in rats. Xenobiotica 2003; 33: 305-21.

19. Kimura H, Liu S, Yamada S, et al. Rapid increase in serum lipid peroxide 4-hydroxynonenal (HNE) through monocyte NADPH oxidase in early endo-toxemia. Free Radic Res 2005; 39: 845-51.

20. Fernandez-Llama P, Jimenez W, Bosch-Marce M, Arroyo $\mathrm{V}$, Nielsen S, Knepper MA. Dysregulation of renal aquaporins and $\mathrm{Na}-\mathrm{Cl}$ cotransporter in $\mathrm{CCl}_{4}$-induced cirrhosis. Kidney Int 2000; 58: 216-28.

21. Anguiano-Robledo L, Reyes-Melchor PA, BobadillaLugo RA, Pérez-Alvarez VM, López-Sánchez P. Renal angiotensin-II receptors expression changes in a model of preeclampsia. Hypertens Pregnancy 2007; 26: 151-61.

22. Devi LA. Heterodimerization of G-protein-coupled receptors: pharmacology, signaling and trafficking. Trends Pharmacol Sci 2001; 22: 532-7.

23. Teichmann A, Gibert A, Lampe A, et al. The specific monomer/dimer equilibrium of the corticotropin-releasing factor receptor type 1 is established in the endoplasmic reticulum. J Biol Chem 2014; 289: 24250-62.

24. AbdAlla S, Lother $\mathrm{H}$, el Missiry $\mathrm{A}$, et al. Dominant negative $\mathrm{AT}_{2}$ receptor oligomers induce $\mathrm{G}$-protein arrest and symptoms of neurodegeneration. J Biol Chem 2009; 284: 6566-74

25. Gill PS, Wilcox CS. NADPH oxidases in the kidney. Antioxid Redox Signal 2006; 8: 1597-607.

26. Miyata K, Rahman M, Shokoji T, et al. Aldosterone stimulates reactive oxygen species production through activation of NADPH oxidase in rat mesangial cells. J Am Soc Nephrol 2005; 16: 2906-12.

27. Liu S, Li Y, Zhao H, et al. Increase in extracellular cross-linking by tissue transglutaminase and reduction in expression of MMP-9 contribute differentially to focal segmental glomerulosclerosis in rats. Mol Cell Biochem 2006; 284: 9-17.

28. Johnson TS, Griffin M, Thomas GL, et al. The role of transglutaminase in the rat subtotal nephrectomy model of renal fibrosis. J Clin Invest 1997; 99: 2950-60.

29. Velez JC. The importance of the intrarenal renin-angiotensin system. Nat Clin Pract Nephrol 2009; 5: 89-100.

30. Miyata N, Park F, Li XF, Cowley AW. Distribution of angiotensin $\mathrm{AT}_{1}$ and $\mathrm{AT}_{2}$ receptor subtypes in the rat kidney. Am J Physiol 1999; 277: F437-46.

31. Ikee R, Kobayashi S, Hemmi N, et al. Involvement of transglutaminase-2 in pathological changes in renal disease. Nephron Clin Pract 2007; 105: c139-46.
32. Shrestha B, Butt I, Da Silva $M$, et al. Upregulation of transglutaminase epsilon (gamma-glutamyl)-lysine in the Fisher-Lewis rat model of chronic allograft nephropathy. Biomed Res Int 2014; 2014: 651608.

33. Huang L, Haylor JL, Hau Z, et al. Transglutaminase inhibition ameliorates experimental diabetic nephropathy. Kidney Int 2009; 76: 383-94.

34. Johnson TS, Fisher M, Haylor JL, et al. Transglutaminase inhibition reduces fibrosis and preserves function in experimental chronic kidney disease. J Am Soc Nephro 2007; 18: 3078-88.

35. Schelling JR. Tissue transglutaminase inhibition as treatment for diabetic glomerular scarring: it's good to be glueless. Kidney Int 2009; 76: 363-5.

36. Fisher M, Jones RA, Huang L, et al. Modulation of tissue transglutaminase in tubular epithelial cells alters extracellular matrix levels: a potential mechanism of tissue scarring. Matrix Biol 2009; 28: 20-31.

37. Asaba K, Tojo A, Onozato ML, et al. Effects of NADPH oxidase inhibitor in diabetic nephropathy. Kidney Int 2005; 67: 1890-8.

38. Li CY, Deng YL, Sun BH. Effects of apocynin and losartan treatment on renal oxidative stress in a rat model of calcium oxalate nephrolithiasis. Int Urol Nephrol 2009; 41: 823-33.

39. Chabrashvili T, Tojo A, Onozato ML, et al. Expression and cellular localization of classic NADPH oxidase subunits in the spontaneously hypertensive rat kidney. Hypertension 2002; 39: 269-74.

40. Wang H, Chen X, Su Y, et al. p47 phox contributes to albuminuria and kidney fibrosis in mice. Kidney Int 2015; 87: 948-62.

41. Liu GC, Fang F, Zhou J, et al. Deletion of p47phox attenuates the progression of diabeticnephropathy and reduces the severity of diabetes in the Akita mouse. Diabetologia 2012; 55: 2522-32. 